\title{
Colitis microscópica, un diagnóstico en aumento
}

\section{Microscopic Cholitis, An Increasingly Frequent Diagnosis}

\author{
Robin German Prieto $0 .{ }^{*}$, Jhon Edison Prieto $0 .{ }^{1}$
}

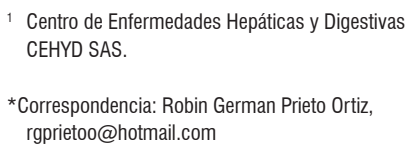

\begin{abstract}
Resumen
Actualmente, la colitis microscópica agrupa tres subgrupos de patologías, las clásicas son la colitis linfocítica $(C L)$ y la colitis colagenosa $(C C)$, que histológicamente se distinguen por la presencia o ausencia de engrosamiento subepitelial; el tercer subgrupo corresponde a la colitis microscópica incompleta (CMI), que incluye a pacientes que no cumplen los criterios clásicos de colitis microscópica, pero que presentan cambios histológicos similares. Aunque se considera una enfermedad con baja prevalencia e incidencia, los estudios presentados en los últimos años evidencian un incremento leve de esta patología. Se han mencionado como factores causales los inmunológicos e infecciosos y se ha relacionado con el consumo de algunos medicamentos y de cigarrillo. Clínicamente se caracteriza por la presencia de diarrea acuosa crónica, que en algunos pacientes puede cursar con períodos de estreñimiento. Los tres subgrupos presentan manifestaciones clínicas similares, por lo que su diagnóstico generalmente es histológico. La colonoscopia con toma de biopsias es el pilar diagnóstico y se debe complementar con hemograma, examen parasitológico, estudios inmunológicos (anticuerpos antinucleares, lgG) y de función tiroidea. El tratamiento se basa en la suspensión de medicamentos relacionados, cambios en los hábitos alimenticios y en el uso de medicamentos, como los esteroides, subsalicilato de bismuto, 5-ASA y colestiramina. En la gran mayoría de los pacientes, la mejoría se logra con un bajo porcentaje de recidivas.
\end{abstract}

\section{Palabras clave \\ Colitis, microscópica, linfocítica, colagenasa, colonoscopia, biopsias.}

\begin{abstract}
Microscopic colitis currently includes three subgroups. The classical ones are lymphocytic colitis and collagenous colitis which are distinguished histologically by the presence or absence of subepithelial thickening. The third subgroup is Incomplete Microscopic Colitis which includes patients who do not meet the classical criteria for Microscopic colitis but who have similar histological changes. Although prevalence and incidence are low, recent studies show that it has become slightly more common. Causative factors mentioned include immunological and infectious issue, and it has been related to some medications and to cigarette smoking. Clinically it is characterized by watery diarrhea which sometimes oscillate with periods of constipation. The three subgroups have similar clinical manifestations, so their diagnoses are usually histological. Colonoscopy with biopsy is the diagnostic pillar, and should be complemented by complete blood count, a parasitological examination, immunological studies (antinuclear antibodies, IgG) and thyroid function. Treatment is based on the suspension of related medications, changes in eating habits, and the use of medications such as steroids, bismuth subsalicylate, 5-ASA and cholestyramine. Improvement is achieved in the vast majority of patients, and recurrences are rare.
\end{abstract}

\section{Keywords}

Colitis, microscopic, lymphocytic, collagenase, colonoscopy, biopsies. 


\section{INTRODUCCIÓN}

La primera publicación de un caso de colitis microscópica se atribuye a Lindstrom, quien, en 1976, informó el caso de una paciente con diarrea crónica acuosa, con biopsias de colon, que evidenció la presencia de un importante depósito de colágeno subepitelial (1). En 1980, Read presentó un trabajo en el que se utilizó por primera vez el término de colitis microscópica (CM) para referirse a pacientes que presentaban diarrea crónica acuosa, con estudios de colon por enema y colonoscopia normales en la mayoría de los casos, pero con biopsias que mostraban alteraciones histológicas (2). A partir de 1993 se propusieron dos subtipos clásicos de CM: la colitis linfocítica (CL) y la colitis colagenosa (CC), que son entidades clínicamente similares, pero que histológicamente se distinguen por la presencia o ausencia de engrosamiento subepitelial, determinado por una banda de colágeno (3-5). En las últimas décadas se han presentado estudios de pacientes que no cumplen los criterios clásicos de CM, pero que presentan otros cambios histológicos similares, por lo cual se acepta universalmente el término de colitis microscópica incompleta (CMI) (6).

En Estados Unidos, la incidencia de CL para el año 2001 fue de 64 casos por cada 100000 personas y la de CC de 36 casos por cada 100000 personas, con una prevalencia del $2 \%$ al $16 \%$ en los pacientes con diarrea crónica (7). En Latinoamérica, los estudios de colitis microscópica son pocos y se realizaron a comienzos de la década pasada, en los que se encontró una prevalencia de hasta el $9 \%$ en los pacientes con diarrea crónica (8-10). En la última década se han presentado algunas revisiones de tema que muestran un leve incremento de esta patología $(5,11-13)$. En nuestro medio no tenemos cifras recientes en relación con esta enfermedad.

\section{DISCUSIÓN}

La CM es un término genérico, que incluye principalmente dos enfermedades que pueden ser de tipo crónico o recidivante y que poseen características histopatológicas similares, como lesiones epiteliales superficiales (leve en la CL y moderada a severa en la CC), distorsión arquitectónica leve o ausente en las criptas y a veces cambios focales similares a los observados en la enfermedad inflamatoria intestinal (EII), como la criptitis y la metaplasia celular $(6,14)$.

La CL se caracteriza por un incremento de los linfocitos intraepiteliales (LIE) (>20 linfocitos/100 células epiteliales), tanto en el epitelio superficial como en el epitelio de las criptas, en una mucosa con un patrón arquitectural normal, en el que, además, se observa daño en el epitelio superficial y un infiltrado inflamatorio mononuclear mixto (plasmocitos, linfocitos) y escasos eosinófilos en la lámina propia, en ausencia de un depósito de colágeno subepitelial $(11,15,16)$. La CC se caracteriza por una banda de colágeno subepitelial engrosada, que por definición mide más de $10 \mu \mathrm{m}$ de grosor. Medir esta banda resulta dispendioso y no es práctico, por lo que está descrito que la clave para el diagnóstico no es solo el aumento del grosor de la banda, sino su patrón de distribución anormal, que se extiende dentro de la lámina propia y envuelve y atrapa a los capilares y fibroblastos $(11,15,17)$.

La CM fue considerada inicialmente una enfermedad rara, hasta 1992 se habían informado apenas 446 casos de CC (18). En los últimos años se ha realizado un mayor número de estudios que evalúan la incidencia y prevalencia, en los que se ha observado importantes variaciones geográficas. Según un metaanálisis, para el año 2015, la incidencia de CC fue 4,14 (IC 95\% 2,89-5,4) por 100000 personasaño y 4,85 (IC 95\% 3,45-6,25) para CL (19). De acuerdo con diversos estudios, la CM es más común en las mujeres, con una relación hombre:mujer que varía de 1:4-1:9 (14, 15). La CC ocurre más frecuentemente en mayores de 50 años, aunque puede presentarse a cualquier edad, en tanto que la CL se presenta en pacientes más jóvenes $(20,21)$.

\section{ETIOLOGÍA}

Existen varias teorías que tratan de explicar la CM, las más comunes hacen referencia a la activación del sistema inmune de la mucosa colónica como respuesta a la exposición a diferentes factores antigénicos luminales, como toxinas, infecciones, ácidos biliares o fármacos (22). Recientemente se ha demostrado la existencia de un antígeno leucocitario humano, que se relaciona directamente con los mecanismos inflamatorios a nivel de la mucosa colónica $(23,24)$.

La posible etiología infecciosa de la $\mathrm{CM}$ se basa en varias observaciones clínicas, que incluyen el desarrollo de CM después de una infección intestinal por Clostridium difficile o la presencia de un mayor número de anticuerpos contra Yersinia enterocolitíca y otros agentes infecciosos en el suero de los pacientes con CC en comparación con los sujetos control; sin embargo, no se ha identificado un patógeno único relacionado con la CM (25-27).

La teoría sobre el efecto potencial de los ácidos biliares en el desarrollo de la CM se basa en modelos experimentales de la enfermedad en animales, la aparición frecuente de diarrea con malabsorción de ácido biliar después de una resección ileal y la demostración de que hay malabsorción en una proporción significativa de pacientes con CM (22, 28-32). No existe evidencia sobre el papel etiológico de los ácidos biliares en la CM y no se ha observado remisión de las lesiones histológicas en los pacientes tratados con agentes secuestrantes de ácidos biliares (33). 
Desde la década de 1990 se ha considerado el uso permanente de algunos medicamentos (Tabla 1) como causante o precipitante de la $\mathrm{CM}(34,35)$. Las guías del Instituto Americano de Gastroenterología para el manejo de la CM consideran varios medicamentos, que incluyen los antiinflamatorios no esteroideos (AINE), la aspirina, los inhibidores de la bomba de protones (IBP), los inhibidores selectivos de la recaptación de serotonina (ISRS), la clozapina y la acarbosa; sin embargo, el grado de relación causa efecto es muy variable de acuerdo con los diversos informes $(32,36,37)$.

Tabla 1. Medicamentos asociados con la colitis microscópica

\begin{tabular}{|c|c|}
\hline Cardiovasculares & $\begin{array}{l}\text { Bloqueantes } \beta \\
\text { Vinburnina (vasodilatador) } \\
\text { Lisinopril } \\
\text { Simvastatina } \\
\text { Antagonista del receptor de la angiotensina II }\end{array}$ \\
\hline Antiplaquetarios & $\begin{array}{l}\text { Ticlopidina } \\
\text { Aspirina }\end{array}$ \\
\hline De acción central & $\begin{array}{l}\text { Paroxetina } \\
\text { Sertralina } \\
\text { Carbamazepina }\end{array}$ \\
\hline Gastrointestinales & $\begin{array}{l}\text { Inhibidores de la bomba de protones } \\
\text { Ranitidina }\end{array}$ \\
\hline Diversos & $\begin{array}{l}\text { AINE } \\
\text { Tardifero (suplemento de hierro) } \\
\text { Bifosfonatos } \\
\text { Flutamida }\end{array}$ \\
\hline
\end{tabular}

De los factores ambientales, fumar es el factor de riesgo mejor documentado para CM. Varios estudios han demostrado que el tabaquismo está asociado con CC y con CL. Se ha observado la aparición de la enfermedad 10 años antes en el grupo de fumadores en comparación con el grupo de no fumadores, pero el tabaquismo no influyó en el curso ulterior de la enfermedad. Se ha implicado también la ingesta de alcohol como factor contribuyente a la génesis de la CM (38).

\section{DIAGNÓSTICO}

La CM se caracteriza clínicamente por cursar con diarrea crónica acuosa sin sangre. Puede ser moderada o severa, con urgencia defecatoria y se han encontrado casos de pacientes con histopatología de CM que son asintomáticos. Casi la mitad de los pacientes con CM cumplen, además, criterios de síndrome de intestino irritable (SII), como dolor y distensión abdominal, y períodos de estreñimiento (39). Las manifestaciones clínicas de la CL y la CC son muy similares, por lo que no es posible diferenciarlas clínicamente (13).
Generalmente, la aparición de los síntomas es paulatina, pero en un $25 \%-40 \%$ de los casos puede ser repentina. La mayoría de los pacientes tienen un episodio único y logran la remisión espontánea hasta en el 10\% de los casos. En cerca de un $30 \%$ de los pacientes se observa un comportamiento crónico recurrente y refractariedad en el tratamiento en hasta el $7 \%$ de los pacientes $(40,41)$.

Como parte del diagnóstico se debe realizar hemograma completo con velocidad de sedimentación globular, química sanguínea, coprocultivos y exámenes parasitológicos, además de estudios de la función tiroidea e inmunológicos (anticuerpos antinucleares, IgG); recientemente se ha indicado la medición de la calprotectina. El estudio diagnóstico más importante es la colonoscopia que, aunque en hasta el $80 \%$ de los casos es informada como normal, permite la toma de biopsias, que se debe realizar en todos los segmentos del colon. El estudio histológico finalmente es el que confirma el diagnóstico de colitis microscópica $(13,42)$.

\section{TRATAMIENTO}

Como parte inicial del tratamiento se debe evaluar la ingesta de medicamentos relacionados con la CM y suspender o modificar la ingesta de los mismos. La suspensión de la ingesta de cafeína y lactosa puede tener algunos beneficios. Cerca de $30 \%$ de los pacientes puede tener una remisión espontánea, por lo que pueden ser tratados sintomáticamente mediante la administración de loperamida. Los medicamentos que se han empleado en el tratamiento de la CM son esteroides (budesónida y prednisona), subsalicilato de bismuto, 5-ASA y colestiramina (agente aglutinante de ácido biliar). De ellos, solo la budesónida cuenta con ensayos aleatorios que demuestran la efectividad en $\mathrm{CL}$ y CC, tanto en la inducción como en el mantenimiento de la remisión clínica. La budesónida es un corticoide localmente activo, que es bien tolerado y tiene poca absorción sistémica. La dosis recomendada es de $9 \mathrm{mg} /$ día durante el primer mes, con disminución a 6 y $3 \mathrm{mg} /$ día en el segundo y tercer mes. Algunos estudios sugieren el uso de los inmunosupresores, como la azatioprina y los agentes antifactor de necrosis tumoral, especialmente en los casos refractarios (42-44).

La CM tiene un pronóstico muy bueno, con remisión espontánea o con una terapia adecuada. Una vez lograda la remisión clínica, el curso es benigno y la mayoría de los pacientes se mantienen asintomáticos a largo plazo. La recidiva ocurre en menos del $30 \%$ de los pacientes y tiene una adecuada respuesta al tratamiento. Se ha informado muy pocos casos que han requerido tratamiento quirúrgico (ileostomía o proctocolectomía total). La CM no se ha relacionado con aumento de la mortalidad y no parece existir un potencial maligno $(13,45)$. 


\section{CONCLUSIONES}

Tanto la colitis linfocítica como la colitis colagenasa son enfermedades cuyo diagnóstico se realiza cada vez más frecuente. Estos diagnósticos deben sospecharse siempre en los pacientes con diarrea crónica, en quienes la realización de colonoscopia con toma seriada de biopsia facilitará el diagnóstico histológico que, junto con la clínica y el hemograma, favorecerán la realización del diagnóstico. No hay consenso en cuanto a la etiología ni a los criterios diagnósticos de estos pacientes. El tratamiento generalmente logra la remisión de la sintomatología, aunque se pueden observar recaídas hasta en casi la tercera parte de estos pacientes. Esta patología, cada vez más frecuentemente informada en la literatura, amerita la realización de estudios que permi$\tan$ establecer su verdadera prevalencia en nuestro medio.

\section{Conflicto de interés}

El presente artículo no representa ningún conflicto de intereses por parte de los autores.

\section{Fuente de financiación}

Los autores.

\section{REFERENCIAS}

1. Lindström CG. 'Collagenous colitis' with watery diarrhoea, a new entity? Pathol Eur. 1976;11(1):87-89.

2. Read NW, Miles CA, Fisher D, Holgate AM, Kime ND, Mitchell MA, et al. Transit of a meal through the stomach, small intestine, and colon in normal subjects and its role in the pathogenesis of diarrhea. Gastroenterology. 1980;79:1276-82. doi: https://doi.org/10.1016/00165085(80)90925-7.

3. Nguyen GC, Smalley WE, Vege SS, Carrasco-Labra A. American Gastroenterological Association Institute Guideline on the Medical Management of Microscopic Colitis. Gastroenterology. 2016;150:242-6. doi: https:// doi.org/10.1053/j.gastro.2015.11.008.

4. Fernández-Bañares F, Casanova MJ, Arguedas Y, Beltrán B, Busquets D, Fernández JM, et al. Current concepts on microscopic colitis: evidence-based statements and recommendations of the Spanish Microscopic Colitis Group. Aliment Pharmacol Ther. 2016;43:400-26. doi: https://doi. org/10.1111/apt.13477.

5. Perez-Manauta J. Colitis microscópica. Rev Gastroenterol Mex. 2011;76(1):72-4.

6. Langner C, Aust D, Ensari A, Villanacci V, Becheanu G, Miehlke S, et al. Histology of microscopic colitis-review with a practical approach for pathologists. Histopathology. 2015;66:613-26. doi: https://doi.org/10.1111/his.12592.
7. Pardi DS, Kelly CP. Microscopic colitis. Gastroenterology. 2011;140:1155. doi: https://doi.org/10.1053/j.gastro.2011.02.003.

8. Coronel F, Sáenz FR, Sáenz FM, Schultz M, Navarrete GC. Colitis microscópica: valor predictivo de la sospecha clínico-endoscópica en nuestro medio. Gastr Latinoam 2005; 16:186-91.

9. Castaño Llano R, Puerta J, Sanin E. Colonoscopía total en pacientes con diarrea crónica: evaluación de una cohorte con colitis linfocítica y colagenosa. Rev Col Gastroenterol. 2002;16:180-9.

10. Valle Mansilla JL, León Barúa R, Recavarren Arce S, Berendson R, Biber Poillevard M. Colitis microscópica en pacientes con diarrea crónica. Rev Gastroenterol Perú. 2002;22:275-8.

11. Melo M, Castilla E. Diagnóstico histológico de colitis microscópica: enfoque práctico. Rev Col Gastroenterol. 2013;28:311-9.

12. Arevalo F, Aragon V, Montes P, Pérez Narrea T, Monge E. Colitis eosinofílica y colitis linfocitaria: ¿diferentes manifestaciones de un mismo proceso en pacientes con diarrea crónica? Rev Gastroenterol Perú. 2013;33:39-42.

13. Bauta J, Pupo A. Colitis microscópica. CCM. 2017;2:526-39.

14. Guagnozzi D, Landolfi S, Vicario M. Towards a new paradigm of microscopic colitis: Incomplete and variant forms. World J Gastroenterol. 2016;22:8459-71. doi: https://doi. org/10.3748/wjg.v22.i38.8459.

15. Montgomery EA, Voltaggio L. Biopsy interpretation of the gastrointestinal tract mucosa. Volume 1, Non-Neoplasia. Philadelphia, PA: Lippincott Williams \& Wilkins. 2012.

16. Lazenby AJ, Yardley JH, Giardiello FM, Jessurun J, Bayless TM. Lymphocytic ("microscopic") colitis: a comparative histopathologic study with particular reference to collagenous colitis. Hum Pathol. 1989;20:18-28. doi: https://doi. org/10.1016/0046-8177(89)90198-6.

17. Mahajan D, Goldblum JR, Xiao SY, Shen B, Liu X. Lymphocytic colitis and collagenous colitis: a review of clinicopathologic features and immunologic abnormalities. Adv Anat Pathol. 2012;19:28-38. doi: https://doi. org/10.1097/PAP.0b013e31823d7705.

18. Bohr J, Tysk C, Eriksson S, Järnerot G. Collagenous colitis in Orebro, Sweden, an epidemiological study 19841993. Gut. 1995;37:394-7. doi: https://doi.org/10.1136/ gut.37.3.394.

19. Tong J, Zheng Q, Zhang C, Lo R, Shen J, Ran Z. Incidence, prevalence, and temporal trends of microscopic colitis: a systematic review and meta-analysis. Am J Gastroenterol. 2015;110:26576. doi: https://doi.org/10.1038/ajg.2014.431.

20. Storr MA. Microscopic colitis: epidemiology, pathophysiology, diagnosis and current management - An update 2013. ISRN Gastroenterology. 2013;2013:352718. doi: http:// dx.doi.org/10.1155/2013/352718.

21. Mohamed N, Marais M, Bezuidenhout J. Microscopic colitis as a missed cause of chronic diarrhea. World J Gastroenterol. 2011;17:1996-2002. doi: https://doi.org/10.3748/wjg. v17.i15.1996. 
22. Pisani LF, Tontini GE, Vecchi M, Pastorelli L. Microscopic colitis: what do we know about pathogenesis? Inflamm Bowel Dis. 2016;22:450-8. Doi: https://doi.org/10.1097/ MIB.0000000000000628.

23. Westerlind H, Mellander MR, Bresso F, Munch A, Bonfiglio F, Assadi G, et al. Dense genotyping of immune-related loci identifies HLA variants associated with increased risk of collagenous colitis. Gut. 2017;66(3):421-8. doi: https:// doi.org/10.1136/gutjnl-2015-309934.

24. Westerlind $\mathrm{H}$, Bonfiglio F, Mellander MR, Hübenthal M, Brynedal B, Björk J, et al. HLA associations distinguish collagenous from lymphocytic colitis. Am J Gastroenterol. 2016;111:1211-3. doi: https://doi.org/10.1038/ajg.2016.215.

25. Walter SA, Munch A, Ost A, Strom M. Anorectal function in patients with collagenous colitis in active and clinically quiescent phase, in comparison with healthy controls. Neurogastroenterol Motil. 2010;22(534-8):e118. doi: https://doi.org/10.1111/j.1365-2982.2010.01472.x.

26. Erim T, Alazmi WM, O’Loughlin CJ, Barkin JS. Collagenous colitis associated with Clostridium difficile: a cause effect? Dig Dis Sci. 2003;48:1374-5.

27. Bohr J, Nordfelth R, Jarnerot G, Tysk C. Yersinia species in collagenous colitis: a serologic study. Scand J Gastroenterol. 2002;37:711-4. doi: https://doi. org/10.1080/00365520212509.

28. Breuer NF, Rampton DS, Tammar A, Murphy GM, Dowling $\mathrm{H}$. Effect of colonic perfusion with sulfated and nonsulfated bile acids on mucosal structure and function in the rat. Gastroenterology. 1983;84:969-77. doi: https://doi. org/10.1016/0016-5085(83)90199-3.

29. Lewis FW, Warren GH, Goff JS. Collagenous colitis with involvement of terminal ileum. Dig Dis Sci. 1991;36:11613. doi: https://doi.org/10.1007/BF01297466.

30. Ingle SB, Adgaonkar BD, Ingle CRH. Microscopic colitis: common cause of unexplained nonbloody diarrhea. World J Gastrointest Pathophysiol. 2014;5:48-53. doi: https://doi. org/10.4291/wjgp.v5.i1.48.

31. Fernandez-Banares F, Esteve M, Salas A, Forné TM, Espinos JC, Martín-Comin J, et al. Bile acid malabsorption in microscopic colitis and in previously unexplained functional chronic diarrhea. Dig Dis Sci. 2001;46:2231-8. doi: https://doi. org/10.1023/A:1011927302076.

32. Lucendo A. Drug exposure and the risk of microscopic colitis: a critical update. Drugs R D. 2017;17:79-89. doi: https://doi.org/10.1007/s40268-016-0171-7.

33. Ung KA, Kilander A, Nilsson O, Abrahamsson H. Longterm course in collagenous colitis and the impact of bile acid malabsorption and bile acid sequestrants on histopathology and clinical features. Scand J Gastroenterol. 2001;36:601-9. doi: https://doi.org/10.1080/003655201750163033.
34. Giardiello FM, Hansen FC 3rd, Lazenby AJ, Hellman DB, Milligan FD, Bayless TM, et al. Collagenous colitis in setting of nonsteroidal antiinflammatory drugs and antibiotics. Dig Dis Sci. 1990;35:257-60. doi: https://doi.org/10.1007/ BF01536772.

35. Tanaka M, Mazzoleni G, Riddell RH. Distribution of collagenous colitis: utility of flexible sigmoidoscopy. Gut. 1992;33:65-70. doi: https://doi.org/10.1136/gut.33.1.65.

36. American Gastroenterological Association. AGA Institute Guideline on the management of microscopic colitis: clinical decision support tool. Gastroenterology. 2016;150:276. doi: https://doi.org/10.1053/j.gastro.2015.11.033.

37. Lucendo AJ, Fernández-Bañares F. Colitis microscópica y exposición a fármacos: una revisión crítica. EII Día. 2015;14:94-104. doi: https://doi.org/10.1016/j. eii.2015.08.004.

38. Bohr J, Wickbom A, Hegedus A, Nyhlin , Hultgren E, Tysk C. Diagnosis and management of microscopic colitis: current perspectives. Clin Exp Gastroenterol. 2014;7:27384. doi: https://doi.org/10.2147/CEG.S63905.

39. Olesen M,Eriksson S, BohrJ,Jarnerot G, Tysk C. Microscopic colitis: a common diarrhoeal disease. An epidemiological study in Orebro, Sweden, 1993-1998. Gut. 2004;53:346-50. doi: https://doi.org/10.1136/gut.2003.014431.

40. Ohlsson B. New insights and challenges in microscopic colitis. Ther Adv Gastroenterol. 2015;8:37-47. doi: https://doi. org/10.1177/1756283X14550134.

41. Thörn M, Sjöberg D, Ekbom A, Holmström T, Larsson M, Nielsen A. Microscopic colitis in Uppsala health region, a population-based prospective study 2005-2009. Scand J Gastroenterol. 2013;48:825-30. doi: https://doi.org/10.31 09/00365521.2013.800993.

42. Villanueva S, Alimi Y. Microscopic colitis (lymphocytic and collagenous), eosinophilic colitis, and celiac disease. Clin Colon Rectal Surg. 2015;28:118-26. doi: https://doi. org/10.1055/s-0035-1549365.

43. Nguyen G, Smalley W, Swaroop S, Carrasco-Labra A. American Gastroenterological Association Institute Guideline on the Medical Management of Microscopic Colitis. Gastroenterology. 2016;150:242-6. doi: https:// doi.org/10.1053/j.gastro.2015.11.008.

44. Gentile N, Abdalla A, Khanna S, Smyrk T, Tremaine W, Faubion W, et al. Outcomes of patients with microscopic colitis treated with corticosteroids: a population-based study. Am J Gastroenterol. 2013;108:256-9. doi: https:// doi.org/10.1038/ajg.2012.416.

45. Tysk C, Wickbom A, Nyhlin N, Erikssonb S, Bohr J. Recent advances in diagnosis and treatment of microscopic colitis. Ann Gastroenterol. 2011;24:1-10. 